\title{
SOME FIXED POINT THEOREMS AND CYCLIC CONTRACTIONS IN DISLOCATED QUASI-METRIC SPACES
}

\author{
Arslan Hojat Ansari and Kastriot Zoto
}

\begin{abstract}
In this paper, we established some common fixed point theorems for types of cyclic contractions in the setting of dislocated quasi-metric spaces. Using the type of contraction defined by C-class functions in [2] and the class of continuous functions $G_{3}$ in [23] we extend, generalize and unify some results in the existing literature.
\end{abstract}

Keywords: cyclic map, cyclical contraction, dislocated quasi-metric, common fixed point

\section{Introduction}

There are a lot of generalizations of the concept of metric space. Some of them are the concept of dislocated metric space and dislocated quasi-metric space which were introduced by Hitzler [6, 7] and F. Zeyada [21]. These metrics have the property " nonzero self-distance" of points and play a very important role not only in topology, but also in other branches of science involving mathematics and, especially, logic programming and electronic engineering. In sequel many researchers have studied, generalized and extended the results on the existence of fixed points and common fixed points, in many directions in dislocated spaces (see e.g. [1, 6, 8, 9, 21, 22, 23, 24]).

In 2003 Kirk et al. [16] introduced cyclic contractions in metric spaces and investigated the existence of proximity points and fixed points for cyclic contraction mappings. Since then, many authors have proved common fixed point theorems for self-mappings satisfying a different type of contractive conditions in some spaces (see results in $[5,11,12,13,14,16]$ ).

In this paper, we introduce some notions of $d_{q}$-cyclic contractions and use the concepts of altering distance function [15], concept of $C$-class functions introduced by Ansari [2] and a class of continuous functions $G_{3}$ and also establish common fixed point theorems for cyclic mappings and cyclic contractions in the framework

Received December 23, 2016; accepted October 23, 2017

2010 Mathematics Subject Classification. 46J15, 47H10, 54H25 
of dislocated quasi-metric spaces. Our main theorems extend, generalize and unify most of the existing metrical fixed point results in literature.

Definition 1.1. $[6,21]$ Let $X$ be a non-empty set and $d: X \times X \rightarrow[0, \infty)$ be a function, called a distance function if for all $x, y, z \in X$, satisfies:

$$
\begin{array}{ll}
d_{1}: & d(x, x)=0 \\
d_{2}: & d(x, y)=d(y, x)=0 \Rightarrow x=y \\
d_{3}: & d(x, y)=d(y, x) \\
d_{4}: & d(x, y) \leq d(x, z)+d(z, y) .
\end{array}
$$

If $d$ satisfies the condition $d_{1}-d_{4}$, then $d$ is called a metric on $X$. If it satisfies the conditions $d_{1}, d_{2}$ and $d_{4}$ it is called a quasi-metric space. If $d$ satisfies conditions $d_{2}, d_{3}$ and $d_{4}$ it is called a dislocated metric (or simply $d$-metric). If $d$ satisfies only $d_{2}$ and $d_{4}$ then $d$ is called a dislocated quasi-metric (or simply $d_{q}$-metric) on $X$.

Definition 1.2. [21] A sequence $\left(x_{n}\right)_{n \in \mathbb{N}}$ in a $d_{q}$-metric space $\left(X, d_{q}\right)$ dislocated quasi-converges (for short, $d_{q}$-converges) to $x \in X$ if $\lim _{n \rightarrow \infty} d_{q}\left(x_{n}, x\right)=\lim _{n \rightarrow \infty} d_{q}\left(x, x_{n}\right)$ $=0$. In this case $x$ is called a $d_{q}-$ limit of $\left(x_{n}\right)_{n \in \mathbb{N}}$ and we write $x_{n} \rightarrow x$.

Definition 1.3. [21] A sequence $\left(x_{n}\right)_{n \in \mathbb{N}}$ in a $d_{q}$-metric space $\left(X, d_{q}\right)$ is said to be Cauchy if for every $\varepsilon>0, \exists n_{0} \in N$ such that $\forall m, n \geq n_{0}, d_{q}\left(x_{m}, x_{n}\right)<\varepsilon$ and $d_{q}\left(x_{n}, x_{m}\right)<\varepsilon$.

Definition 1.4. [21] A $d_{q}$-metric space $\left(X, d_{q}\right)$ is complete if every Cauchy sequence is $d_{q}$-convergent in $X$.

Example 1.1. Let $X=[0,1]$ and $d_{q}(x, y)=\max \{x, y\}$. Then the pair $\left(X, d_{q}\right)$ is a dislocated metric space, but it is not a metric space.

Lemma 1.1. [21] Every subsequence of $d_{q}$-convergent sequence to a point $x_{0}$ is $d_{q}$-convergent to $x_{0}$.

Definition 1.5. [21] Let $\left(X, d_{q}\right)$ be a $d_{q}$-metric space. A mapping $T: X \rightarrow X$ is called contraction if there exists $0 \leq \lambda<1$ such that $d_{q}(T x, T y) \leq \lambda d_{q}(x, y)$ for all $x, y \in X$.

Lemma 1.2. [21] The $d_{q}$-limit point in a $d_{q}$-metric space is unique.

Let $K$ and $H$ be nonempty subsets of a metric space $(X, d)$. A map $T: K \cup H \rightarrow$ $K \cup H$ is called cyclic map if $T(K) \subseteq H$ and $T(H) \subseteq K$.

Definition 1.6. [16] Let $K$ and $H$ be nonempty subsets of a metric space $(X, d)$. A cyclic map $T: K \cup H \rightarrow K \cup H$ is said to be a cyclic contraction if there exists $k \in(0,1)$ such that $d(T x, T y) \leq k d(x, y)$ for all $x \in K$ and $y \in H$. 
Definition 1.7. [10] Let $H$ and $K$ be nonempty subsets of a metric space $(X, d)$. A cyclic map $T: K \cup H \rightarrow K \cup H$ is called a Kannan type cyclic contraction if there exists $k \in\left(0, \frac{1}{2}\right)$ such that $d(T x, T y) \leq k[d(T x, x)+d(T y, y)]$ for all $x \in K$ and $y \in H$.

In [10] Karapinar et al. has been shown that the Kannan-type cyclic contraction and cyclic contraction are independent of each other.

Definition 1.8. [10] Let $K$ and $H$ be nonempty subsets of a metric space $(X, d)$. A cyclic map $T: K \cup H \rightarrow K \cup H$ is called a Chatterjee-type cyclic contraction if there exists $k \in\left(0, \frac{1}{2}\right)$ such that $d(T x, T y) \leq k \max [d(x, y), d(T x, x), d(T y, y)]$ for all $x \in K$ and $y \in H$.

Definition 1.9. [16] Let $K$ and $H$ be nonempty subsets of a dislocated metric space $(X, d)$. A cyclic map $T: K \cup H \rightarrow K \cup H$ is called a $d$-cyclic contraction if there exists $k \in(0,1)$ such that $d(T x, T y) \leq k d(x, y)$ for all $x \in K$ and $y \in H$.

Example 1.2. $[22]$ Let $X=[0,1]$ and $d(x, y)=|x-y|+3|x|+3|y|$. Then $(X, d)$ is a dislocated metric space, but not a metric space. Let $K=H=[0,1]$ and define $T: K \cup H \rightarrow K \cup H$ by $T x=\frac{1}{3}$ for $x=1$ and $T x=1$ for $x \in[0,1)$. Then $T$ is a $d$ cyclic contraction in the dislocated metric space $(X, d)$. We note that in the usual metric $d(x, y)=|x-y|$ the self map $T$ is not cyclic contraction because for $x=\frac{11}{12}$ and $y=1$ the cyclic contraction fails.

Hence the class of $d$-cyclical contractions in dislocated metric space is larger than the class of cyclical contractions in usual metric.

Definition 1.10. [22] Let $K$ and $H$ be nonempty subsets of a dislocated quasimetric space $\left(X, d_{q}\right)$. A cyclic map $T: K \cup H \rightarrow K \cup H$ is called a Geraghty-type $d_{q^{-}}$ cyclic contraction if there exists $\beta \in S$ such that $d_{q}(T x, T y) \leq \beta\left(d_{q}(x, y)\right) d_{q}(x, y)$ for all $x \in K$ and $y \in H$.

In 2014 the concept of $C$-class functions (see Definition 1.11) was introduced by Ansari [2], for example see numbers (1), (2), (9) and (15) from Example 1.3. Also see [8] and [3].

Definition 1.11. [2] A continuous function $F:[0, \infty) \times[0, \infty) \rightarrow \mathbb{R}$ is called $C$-function if for any $s, t \in[0, \infty)$, the following conditions hold:

(1) $F(s, t) \leq s$;

(2) $F(s, t)=s$ implies that either $s=0$ or $t=0$.

The letter $\mathcal{C}$ will denote the class of all $C$-functions.

PROBLEM: this is an open problem, we can say that for all $F \in \mathcal{C}, F(0,0)=0$.

Example 1.3. [2] The following examples show that the class $\mathcal{C}$ is nonempty. 
1. $F(s, t)=s-t$.

2. $F(s, t)=m s, 0<m<1$.

3. $F(s, t)=\frac{s}{(1+t)^{r}}$ for some $r \in(0, \infty)$.

4. $F(s, t)=\log \left(t+a^{s}\right) /(1+t)$, for some $a>1$.

5. $F(s, t)=\ln \left(1+a^{s}\right) / 2$, for $a>e$. Indeed $F(s, 1)=s$ implies that $s=0$.

6. $F(s, t)=(s+l)^{\left(1 /(1+t)^{r}\right)}-l, l>1$, for $r \in(0, \infty)$.

7. $F(s, t)=s \log _{t+a} a$, for $a>1$.

8. $F(s, t)=s-\left(\frac{1+s}{2+s}\right)\left(\frac{t}{1+t}\right)$.

9. $F(s, t)=s \beta(s)$, where $\beta:[0, \infty) \rightarrow[0,1)$.

10. $F(s, t)=s-\frac{t}{k+t}$.

11. $F(s, t)=s-\varphi(s)$, where $\varphi:[0, \infty) \rightarrow[0, \infty)$ is a continuous function such that $\varphi(t)=0$ if and only if $t=0$.

12. $F(s, t)=\operatorname{sh}(s, t)$, where $h:[0, \infty) \times[0, \infty) \rightarrow[0, \infty)$ is a continuous function such that $h(t, s)<1$ for all $t, s>0$.

13. $F(s, t)=s-\left(\frac{2+t}{1+t}\right) t$.

14. $F(s, t)=\sqrt[n]{\ln \left(1+s^{n}\right)}$.

15. $F(s, t)=\phi(s)$, where $\phi:[0, \infty) \rightarrow[0, \infty)$ is an upper semicontinuous function such that $\phi(0)=0$ and $\phi(t)<t$ for $t>0$.

16. $F(s, t)=\frac{s}{(1+s)^{r}} ; r \in(0, \infty)$.

Definition 1.12. [15] A function $\psi:[0, \infty) \rightarrow[0, \infty)$ is called an altering distance function if the following properties are satisfied:

(i) $\psi$ is non-decreasing and continuous,

(ii) $\psi(t)=0$ if and only if $t=0$.

We denote altering distance functions as $\Psi$.

Definition 1.13. [2] An ultra altering distance function is a continuous, nondecreasing mapping $\varphi:[0, \infty) \rightarrow[0, \infty)$ such that $\varphi(t)>0, t>0$.

We denote ultra altering distance functions as $\Phi_{u}$. 


\section{Main results}

Lemma 2.1. Let $\left(X, d_{q}\right)$ be a $d_{q}$-metric space and let $\left\{x_{n}\right\}$ be a sequence in $X$ such that

$$
\lim _{n \rightarrow \infty} d_{q}\left(x_{n+1}, x_{n}\right)=\lim _{n \rightarrow \infty} d_{q}\left(x_{n}, x_{n+1}\right)=0 .
$$

If $\left\{x_{n}\right\}$ is not a Cauchy sequence, then there exist $\varepsilon>0$ and two subsequences $\left\{x_{m_{k}}\right\}$ and $\left\{x_{n_{k}}\right\}$ of $\left\{x_{n}\right\}_{n \in N}$ with $n_{k} \geq m_{k} \geq k$, (positive integers) such that the following four sequences tend to $\varepsilon$ for $k \rightarrow \infty$ :

$$
d_{q}\left(x_{m_{k}}, x_{n_{k}}\right), \quad d_{q}\left(x_{m_{k}}, x_{n_{k}+1}\right), \quad d_{q}\left(x_{m_{k}+1}, x_{n_{k}}\right), \quad d_{q}\left(x_{m_{k}+1}, x_{n_{k}+1}\right) .
$$

Proof. If suppose that $\left\{x_{n}\right\}$ is a sequence in $X$ satisfying condition (2.1) which is not Cauchy then there exist $\varepsilon>0$ for which we can find subsequences $\left\{x_{m_{k}}\right\}$ and $\left\{x_{n_{k}}\right\}$ of $\left\{x_{n}\right\}_{n \in N}$ such that $n_{k}$ is the smallest index for which

$$
n_{k} \geq m_{k} \geq k, \quad d_{q}\left(x_{m_{k}}, x_{n_{k}}\right) \geq \varepsilon, \quad d_{q}\left(x_{m_{k}}, x_{n_{k}-1}\right)<\varepsilon .
$$

Then from triangular inequality,

$$
\begin{aligned}
\varepsilon & \leq d_{q}\left(x_{m_{k}}, x_{n_{k}}\right) \leq d_{q}\left(x_{m_{k}}, x_{n_{k}-1}\right)+d_{q}\left(x_{n_{k}-1}, x_{n_{k}}\right) \\
& \leq \varepsilon+d_{q}\left(x_{n_{k}-1}, x_{n_{k}}\right)
\end{aligned}
$$

Taking the limit as $k \rightarrow \infty$ in (2.3), using the condition (2.4) we conclude that

$$
\lim _{k \rightarrow \infty} d_{q}\left(x_{m_{k}}, x_{n_{k}}\right)=\varepsilon
$$

Again

$$
\begin{aligned}
\varepsilon & \leq d_{q}\left(x_{m_{k}}, x_{n_{k}}\right) \leq d_{q}\left(x_{m_{k}}, x_{n_{k}+1}\right)+d_{q}\left(x_{n_{k}+1}, x_{n_{k}}\right) \\
& \leq d_{q}\left(x_{m_{k}}, x_{n_{k}}\right)+d_{q}\left(x_{n_{k}}, x_{n_{k}+1}\right)+d_{q}\left(x_{n_{k}+1}, x_{n_{k}}\right)
\end{aligned}
$$

Taking the limit as $k \rightarrow \infty$ in (2.5), using the condition (2.1) and the result (2.4) we conclude that

$$
\lim _{k \rightarrow \infty} d_{q}\left(x_{m_{k}}, x_{n_{k}+1}\right)=\varepsilon
$$

Further

$$
\begin{aligned}
\varepsilon & \leq d_{q}\left(x_{m_{k}}, x_{n_{k}}\right) \leq d_{q}\left(x_{m_{k}}, x_{m_{k}+1}\right)+d_{q}\left(x_{m_{k}+1}, x_{n_{k}}\right) \\
& \leq d_{q}\left(x_{m_{k}}, x_{m_{k}+1}\right)+d_{q}\left(x_{m_{k}+1}, x_{m_{k}}\right)+d_{q}\left(x_{m_{k}}, x_{n_{k}}\right) .
\end{aligned}
$$

In the two inequalities above the taking limit as $k \rightarrow \infty$ and using the condition (2.1) and the results (2.4) we obtain:

$$
\lim _{k \rightarrow \infty} d_{q}\left(x_{m_{k}+1}, x_{n_{k}}\right)=\varepsilon
$$


By triangular inequality, we have

$$
\begin{aligned}
d_{q}\left(x_{m_{k}}, x_{n_{k}}\right) \leq & d_{q}\left(x_{m_{k}}, x_{m_{k}+1}\right)+d_{q}\left(x_{m_{k}+1}, x_{n_{k}+1}\right)+d_{q}\left(x_{n_{k}+1}, x_{n_{k}}\right) \\
\leq & d_{q}\left(x_{m_{k}}, x_{m_{k}+1}\right)+d_{q}\left(x_{m_{k}+1}, x_{n_{k}}\right) \\
& +d_{q}\left(x_{n_{k}}, x_{n_{k}+1}\right)+d_{q}\left(x_{n_{k}+1}, x_{n_{k}}\right) .
\end{aligned}
$$

In the above inequality, taking the limit as $k \rightarrow \infty$ and using the condition (2.1) and the results $(2.4),(2.7)$ we obtain

$$
\lim _{k \rightarrow \infty} d_{q}\left(x_{m_{k}+1}, x_{n_{k}+1}\right)=\varepsilon,
$$

this completes the proof.

Theorem 2.1. Let $K$ and $H$ be nonempty subsets of a complete dislocated quasimetric space $\left(X, d_{q}\right)$. Let $T: K \cup H \rightarrow K \cup H$ be a cyclic mapping that satisfies the condition

$$
\begin{array}{r}
d_{q}(T x, T y) \leq F\left(\max \left\{d_{q}(x, y), d_{q}(x, T x), d_{q}(y, T y)\right\},\right. \\
\left.\varphi\left(\max \left\{d_{q}(x, y), d_{q}(x, T x), d_{q}(y, T y)\right\}\right)\right)
\end{array}
$$

for all $x \in A$ and $y \in B$ and $F \in C, \varphi \in \Phi_{u}$. Then, $T$ has a unique fixed point in $K \cap H$.

Proof. Taking a point $x \in K$ and using (2.9), we have

$$
\begin{aligned}
d_{q}\left(T^{2} x, T x\right)= & d_{q}(T(T x), T x) \\
\leq & F\left(\max \left\{d_{q}(T x, x), d_{q}\left(T x, T^{2} x\right), d_{q}(x, T x)\right\}\right. \\
& \left.\varphi\left(\max \left\{d_{q}(T x, x), d_{q}\left(T x, T^{2} x\right), d_{q}(x, T x)\right\}\right)\right) \\
\leq & \max \left\{d_{q}(T x, x), d_{q}\left(T x, T^{2} x\right), d_{q}(x, T x)\right\} \\
= & \max \left\{d_{q}(T x, x), d_{q}(x, T x)\right\} .
\end{aligned}
$$

In the same way we have,

$$
\begin{aligned}
d_{q}\left(T x, T^{2} x,\right)= & d_{q}\left(T x, T^{2} x,\right) \\
\leq & F\left(\max \left\{d_{q}(x, T x) d_{q}(x, T x), d_{q}\left(T x, T^{2} x\right)\right\},\right. \\
& \left.\varphi\left(\max \left\{d_{q}(x, T x), d_{q}(x, T x), d_{q}\left(T x, T^{2} x\right)\right\}\right)\right) \\
\leq & \max \left\{d_{q}(x, T x), d_{q}(x, T x), d_{q}\left(T x, T^{2} x\right)\right\} \\
= & \max \left\{d_{q}(T x, x)\right\} \\
\leq & \max \left\{d_{q}(T x, x), d_{q}(x, T x)\right\},
\end{aligned}
$$

so,

$$
\max \left\{d_{q}\left(T^{2} x, T x\right), d_{q}\left(T x, T^{2} x\right)\right\} \leq \max \left\{d_{q}(T x, x), d_{q}(x, T x)\right\}
$$


Using (2.9) and (2.10) we get,

(2.11) $\max \left\{d_{q}\left(T^{3} x, T^{2} x\right), d_{q}\left(T^{2} x, T^{3} x\right)\right\} \leq \max \left\{d_{q}\left(T^{2} x, T x\right), d_{q}\left(T x, T^{2} x\right)\right\}$.

Inductively, using this process for all $n \in N$ we have

$$
\begin{array}{r}
\max \left\{d_{q}\left(T^{n+1} x, T^{n} x\right), d_{q}\left(T^{n} x, T^{n+1} x\right)\right\} \leq \max \left\{d_{q}\left(T^{n} x, T^{n-1} x\right),\right. \\
\left.d_{q}\left(T^{n-1} x, T^{n} x\right)\right\} .
\end{array}
$$

The sequence $\left\{\max \left\{d_{q}\left(T^{n+1} x, T^{n} x\right), d_{q}\left(T^{n} x, T^{n+1} x\right)\right\}\right\}$ is decreasing, so it tends to $r \geq 0$ now with $n \rightarrow \infty$ in the above and using (2.9) we have

$$
r \leq F(r, \varphi(r))
$$

So, $r=0$ or $\varphi(r)=0$, therefore $\lim _{n \rightarrow \infty} d_{q}\left(T^{n} x, T^{n-1} x\right)=0$ and $\lim _{n \rightarrow \infty} d_{q}\left(T^{n-1} x, T^{n} x\right)$ $=0$. Now, we shall prove that $\left\{x_{n}\right\}$ is a Cauchy sequence, If $\left\{x_{n}\right\}$ is not a Cauchy sequence, then by Lemma 2.1 there exist $\varepsilon>0$ and two sequences $\left\{m_{k}\right\}$ and $\left\{n_{k}\right\}$ of positive integers such that the following four sequences tend to $\varepsilon$ when $k \rightarrow \infty$,

$$
d_{q}\left(x_{m_{k}}, x_{n_{k}}\right), \quad d_{q}\left(x_{m_{k}}, x_{n_{k}+1}\right), \quad d_{q}\left(x_{m_{k}+1}, x_{n_{k}}\right), \quad d_{q}\left(x_{m_{k}+1}, x_{n_{k}+1}\right) .
$$

So

$$
\begin{aligned}
d_{q}\left(x_{m_{k}+1}, x_{n_{k}+1}\right)= & d_{q}\left(T x_{m_{k}}, T x_{n_{k}}\right) \\
\leq & F\left(\max \left\{d_{q}\left(x_{m_{k}}, x_{n_{k}}\right), d_{q}\left(x_{n_{k}}, T x_{n_{k}}\right), d_{q}\left(x_{m_{k}}, T x_{m_{k}}\right)\right\}\right. \\
& \left.\varphi\left(\max \left\{d_{q}\left(x_{m_{k}}, x_{n_{k}}\right), d_{q}\left(x_{n_{k}}, T x_{n_{k}}\right), d_{q}\left(x_{m_{k}}, T x_{m_{k}}\right)\right\}\right)\right) \\
= & F\left(\max \left\{d_{q}\left(x_{m_{k}}, x_{n_{k}}\right), d_{q}\left(x_{n_{k}}, x_{n_{k}+1}\right), d_{q}\left(x_{m_{k}}, x_{m_{k}+1}\right)\right\},\right. \\
& \left.\varphi\left(\max \left\{d_{q}\left(x_{m_{k}}, x_{n_{k}}\right), d_{q}\left(x_{n_{k}}, x_{n_{k}+1}\right), d_{q}\left(x_{m_{k}}, x_{m_{k}+1}\right)\right\}\right)\right) .
\end{aligned}
$$

Letting $k \rightarrow \infty$, we get

$$
\varepsilon \leq F(\varepsilon, \varphi(\varepsilon)) .
$$

So, $\varepsilon=0$ or $\varphi(\varepsilon)=0$, that is; $\varepsilon=0$. Thus $\left(T^{n} x\right)$ is a Cauchy sequence. Since $\left(X, d_{q}\right)$ is complete, we have $\left(T^{n} x\right) d_{q}$-converges to some $z \in X$. We note that $\left(T^{2 n} x\right)$ is a sequence in $K$ and $\left(T^{2 n-1} x\right)$ is a sequence in $H$ in a way that both sequences tend to the same limit $z$.

Since $K$ and $H$ are closed then we have that $z \in K \cap H$. Hence $K \cap H \neq \varnothing$.

We claim that $T z=z$.

Considering the condition (2.9) we have:

$$
\begin{aligned}
d_{q}\left(T^{2 n} x, T z\right) & =d_{q}\left(T T^{2 n-1} x, T z\right) \\
\leq & F\left(\max \left\{d_{q}\left(T^{2 n-1} x, z\right), d_{q}\left(T^{2 n-1} x, T^{2 n} x\right), d_{q}(z, T z)\right\}\right. \\
& \left., \varphi \max \left\{d_{q}\left(T^{2 n-1} x, z\right), d_{q}\left(T^{2 n-1} x, T^{2 n} x\right), d_{q}(z, T z)\right\}\right) .
\end{aligned}
$$


By $n \rightarrow \infty$ in the above inequality, we have

$$
d_{q}(z, T z) \leq F\left(d_{q}(z, T z), \varphi\left(d_{q}(z, T z)\right)\right) .
$$

So, $d_{q}(z, T z)=0$ or $\varphi\left(d_{q}(z, T z)\right)=0$. This implies that $d_{q}(z, T z)=0$.

Similarly, considering (2.9) we have,

$$
\begin{aligned}
d_{q}\left(T z, T^{2 n} x\right)= & d_{q}\left(T z, T T^{2 n-1} x\right) \\
\leq & F\left(\max \left\{d_{q}\left(z, T^{2 n-1} x\right), d_{q}(z, T z), d_{q}\left(T^{2 n-1} x, T^{2 n} x\right)\right\}\right. \\
& \left., \varphi \max \left\{d_{q}\left(z, T^{2 n-1} x\right), d_{q}(z, T z), d_{q}\left(T^{2 n-1} x, T^{2 n} x\right)\right\}\right) .
\end{aligned}
$$

Similarly, by $n \rightarrow \infty$ above we obtain $d_{q}(T z, z)=0$.

Hence $d_{q}(z, T z)=d_{q}(T z, z)=0$, so $T z=z$ and $z$ is a fixed point of $T$.

We shall prove that $z$ is a unique fixed point of $T$. Clearly, from (2.9) if $u$ and $v$ are the fixed points of $T$ we have $d_{q}(u, u)=0, d_{q}(v, v)=0$.

Then we have,

$$
\begin{aligned}
d_{q}(u, v)= & d_{q}(T u, T v) \\
\leq & F\left(\max \left\{d_{q}(u, v), d_{q}(u, u), d_{q}(v, v)\right\},\right. \\
& \left.\varphi\left(\max \left\{d_{q}(u, v), d_{q}(u, u), d_{q}(v, v)\right\}\right)\right) \\
= & F\left(d_{q}(u, v), \varphi\left(d_{q}(u, v)\right)\right),
\end{aligned}
$$

so, $d_{q}(u, v)=0$ or $\varphi\left(d_{q}(u, v)\right)=0$, therefore $d_{q}(u, v)=0$. Similarly, we can prove that $d_{q}(v, u)=0$, now from $d_{q}(u, v)=d_{q}(v, u)=0$ we deduce that $u=v$. Hence the proof is completed.

Theorem 2.2. Let $K$ and $H$ be nonempty closed subsets of a complete dislocated quasi-metric space $\left(X, d_{q}\right)$ and $T: K \cup H \rightarrow K \cup H$ be a cyclic mapping that satisfies the condition:

$$
d_{q}(T x, T y) \leq F\left(d_{q}(x, y), \varphi\left(d_{q}(x, y)\right)\right)
$$

for all $x \in K$ and $y \in H$ where $F \in C, \varphi \in \Phi_{u}$.Then $T$ has a unique fixed point in $K \cap H$.

Proof. If for all $a, b \in[0, \infty)$ with $a \leq b$ we have that $F(a, \varphi(a)) \leq F(b, \varphi(b))$. In this case the theorem is a corollary of Theorem 2.1, because

$$
\begin{aligned}
d_{q}(T x, T y) & \leq F\left(d_{q}(x, y), \varphi\left(d_{q}(x, y)\right)\right) \\
& \leq F\left(\max \left\{d_{q}(x, y), d_{q}(x, T x), d_{q}(y, T y)\right\},\right. \\
& \left.\varphi\left(\max \left\{d_{q}(x, y), d_{q}(x, T x), d_{q}(y, T y)\right\}\right)\right) .
\end{aligned}
$$

Now assume that there is $a<b$ such that $F(a, \varphi(a))>F(b, \varphi(b))$. In this case, fix a point $x \in A$. If $T^{n} x=T^{n+1} x$ for some $n \in N$, then $T^{n+1} x=T^{n+2} x$ and so 
$\left(T^{n} x\right)$ converges to some $z \in X$. Suppose that $T^{n} x \neq T^{n+1} x$. Using the condition (2.15) we have,

$$
d\left(T^{n+1} x, T^{n} x\right) \leq F\left(d\left(T^{n} x, T^{n-1} x\right), \varphi\left(d\left(T^{n} x, T^{n-1} x\right)\right)\right) \leq d\left(T^{n} x, T^{n-1} x\right) .
$$

Thus the sequence $d\left(T^{n+1} x, T^{n} x\right)$ is decreasing and bounded from below, thus it converges to some $z \geq 0$, then from (2.15) taking the limit as $n \rightarrow \infty$, we get

$$
r \leq F(r, \varphi(r)) .
$$

So, $r=0$ or $\varphi(r)=0$, therefore $\lim _{n \rightarrow \infty} d_{q}\left(T^{n} x, T^{n-1} x\right)=0$. In a similar way we obtain $\lim _{n \rightarrow \infty} d_{q}\left(T^{n-1} x, T^{n} x\right)=0$.

Now, we shall prove that $\left\{x_{n}\right\}$ is a Cauchy sequence, If $\left\{x_{n}\right\}$ is not a Cauchy sequence, then there exist $\varepsilon>0$ and two sequences $\left\{m_{k}\right\}$ and $\left\{n_{k}\right\}$ of positive integers such that the following four sequences tend to $\varepsilon$ when $k \rightarrow \infty$,

$$
d_{q}\left(x_{m_{k}}, x_{n_{k}}\right), \quad d_{q}\left(x_{m_{k}}, x_{n_{k}+1}\right), \quad d_{q}\left(x_{m_{k}+1}, x_{n_{k}}\right), \quad d_{q}\left(x_{m_{k}+1}, x_{n_{k}+1}\right),
$$

SO

$$
d_{q}\left(x_{m_{k}+1}, x_{n_{k}+1}\right) \leq F\left(d_{q}\left(x_{m_{k}}, x_{n_{k}}\right), \varphi\left(d_{q}\left(x_{m_{k}}, x_{n_{k}}\right)\right)\right)
$$

then from(2.16) taking the limit as $k \rightarrow \infty$, we get

$$
\varepsilon \leq F(\varepsilon, \varphi(\varepsilon)) .
$$

So, $\varepsilon=0$ or $\varphi(\varepsilon)=0$, that is; $\varepsilon=0$, our supposition fail from this contradiction. Hence, this proves that $\left(T^{n} x\right)$ is a Cauchy sequence. Since $\left(X, d_{q}\right)$ is complete, we

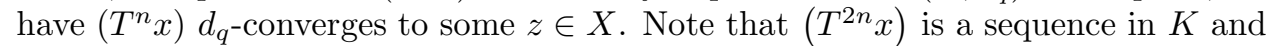
$\left(T^{2 n-1} x\right)$ is a sequence in $H$ in a way that both sequences tend to the same limit $z \in K \cap H$

Considering the condition (2.15) we have,

$$
\begin{aligned}
d_{q}(z, T z) & \leq d_{q}\left(z, T^{2 n} x\right)+d_{q}\left(T^{2 n} x, T z\right) \\
& \leq d_{q}\left(z, T^{2 n} x\right)+F\left(d_{q}\left(T^{2 n-1} x, z\right), \varphi\left(d_{q}\left(T^{2 n-1} x, z\right)\right)\right) .
\end{aligned}
$$

By $n \rightarrow \infty$ in $(2.17)$, we have $d_{q}(T z, z)=0$ and, similarly, we have $d_{q}(z, T z)=0$ as a result $T z=z$.

Uniqueness: Let $u$ and $v$ be two fixed points of $T$. Then

$$
\begin{aligned}
d_{q}(u, v) & =d_{q}(T u, T v) \\
& \leq F\left(d_{q}(u, v), \varphi\left(d_{q}(u, v)\right)\right) \\
& \leq d_{q}(u, v), \\
d_{q}(v, u) & =d_{q}(T v, T u) \\
& \leq F\left(d_{q}(v, u), \varphi\left(d_{q}(v, u)\right)\right) \\
& \leq d_{q}(v, u),
\end{aligned}
$$

so, $d_{q}(u, v)=0$ or $\varphi\left(d_{q}(u, v)\right)=0$, and $d_{q}(v, u)=0$ or $\varphi\left(d_{q}(v, u)\right)=0$, so from those we get $d_{q}(u, v)=d_{q}(v, u)=0$ and also, by property $d_{2}$ we have $u=v$. 
Example 2.1. Let $X=[0,1]$ and $T: X \rightarrow X$ be given as $T(x)=\frac{x}{8}$. Let $K=H=$ $[0,1]$. Define the function $d_{q}: X \times X \rightarrow[0, \infty)$ by $d(x, y)=\max \{x, y\}$. The function $F(s, t)=\frac{s}{1+t}:[0, \infty) \rightarrow[0,1)$ and $\varphi(t)=1$. Considering all the cases and the general cases if $x \leq y, \forall x, y \in X$

$$
\begin{aligned}
d_{q}(T x, T y) & =d_{q}\left(\frac{x}{8}, \frac{y}{8}\right)=\max \left\{\frac{x}{8}, \frac{y}{8}\right\} \\
& =\frac{y}{8} \leq \frac{y}{1+1}=\frac{\max \{x, y\}}{1+\varphi(\max \{x, y\})} \\
& =\frac{d_{q}(x, y)}{1+\varphi\left(d_{q}(x, y)\right)}
\end{aligned}
$$

Clearly, all conditions of Theorem 2.2 are satisfied and $x=0$ is a unique fixed point of $T$.

We consider the set $G_{3}$ of all continuous functions [foe some examples for these functions see $[23]] g:[0, \infty) \times[0, \infty) \times[0, \infty) \rightarrow[0, \infty)$ with the following properties:

(i) $g$ is non-decreasing with respect to each variable.

(ii) $g(t, t, t) \leq t$, for $t \in[0, \infty)$.

Theorem 2.3. Let $K$ and $H$ be nonempty closed subsets of a complete dislocated quasi-metric space $\left(X, d_{q}\right)$ and $T: K \cup H \rightarrow K \cup H$ be a cyclic mapping that satisfies the following condition:

$$
\begin{aligned}
& d(T x, T y) \leq F( g\left[d_{q}(x, y), d_{q}(x, T x), d_{q}(y, T y)\right], \\
&\left.\varphi\left(g\left[d_{q}(x, y), d_{q}(x, T x), d_{q}(y, T y)\right]\right)\right)
\end{aligned}
$$

for all $x \in K$ and $y \in H$, where $F \in C, \varphi \in \Phi_{u}, g \in G_{3}$. Then $T$ has a unique fixed point in $K \cap H$.

Proof. Let $x$ be a fixed point in $X$. By condition (2.18) and the properties of $g$ we have,

$$
\begin{aligned}
d_{q}\left(T^{2} x, T x\right) \leq & F\left(g\left[d_{q}(T x, x), d_{q}\left(T x, T^{2} x\right), d_{q}(x, T x)\right]\right. \\
& \left.\varphi\left(g\left[d_{q}(T x, x), d_{q}\left(T x, T^{2} x\right), d_{q}(x, T x)\right]\right)\right) \\
\leq & d_{q}(T x, x),
\end{aligned}
$$

similarly, we have

$$
\begin{aligned}
d_{q}\left(T^{3} x, T^{2} x\right) \leq & F\left(g\left[d_{q}\left(T^{2} x, T x\right), d_{q}\left(T^{3} x, T^{2} x\right), d_{q}\left(T x, T^{2} x\right)\right]\right. \\
& \left.\varphi\left(g\left[d_{q}\left(T^{2} x, T x\right), d_{q}\left(T^{3} x, T^{2} x\right), d_{q}\left(T x, T^{2} x\right)\right]\right)\right) \\
\leq & d_{q}\left(T^{2} x, T x\right) .
\end{aligned}
$$

Generally, from the above inequalities we have,

$$
d_{q}\left(T^{n+1} x, T^{n} x\right) \leq d_{q}\left(T^{n} x, T^{n-1} x\right)
$$


for $n \in N$.

Thus the sequence $\left\{d_{q}\left(T^{n+1} x, T^{n} x\right)\right\}$ is decreasing and bounded from below, thus it converges to some $z \geq 0$, then from (2.15) taking the limit as $n \rightarrow \infty$, we get

$$
r \leq F(r, \varphi(r)) .
$$

so, $r=0$ or $\varphi(r)=0$, therefore $\lim _{n \rightarrow \infty} d_{q}\left(T^{n} x, T^{n-1} x\right)=0$. In a similar way we obtain $\lim _{n \rightarrow \infty} d_{q}\left(T^{n-1} x, T^{n} x\right)=0$.

Easily, as in the above theorems, we can show that the sequence $\left(T^{n} x\right)$ is a Cauchy sequence in a complete dislocated quasi-metric space $\left(X, d_{q}\right)$. So there exists $z \in X$ such that $\left(T^{n} x\right)$ dislocated quasi converges to $z$. Note that $\left(T^{2 n} x\right)$ is a sequence in $K$ and $\left(T^{2 n-1} x\right)$ is a sequence in $H$ in a way that both sequences tend to the same limit $z \in K \cap H$. To prove that $z$ is a fixed point of $T$ we reuse the contractive condition (2.18),

$$
\begin{aligned}
d_{q}(z, T z) \leq & d_{q}\left(z, T^{2 n} x\right)+d_{q}\left(T^{2 n} x, T z\right) \\
\leq & d_{q}\left(z, T^{2 n} x\right)+F\left(g\left[d_{q}\left(T^{2 n-1} x, z\right), d_{q}\left(T^{2 n-1} x, T^{2 n} x\right), d_{q}(z, T z)\right],\right. \\
& \left.\varphi g\left[d_{q}\left(T^{2 n-1} x, z\right), d_{q}\left(T^{2 n-1} x, T^{2 n} x\right), d_{q}(z, T z)\right]\right) .
\end{aligned}
$$

In this inequality passing the limit as $n \rightarrow \infty$ and since $g$ is non-decreasing and continuous we get,

$$
d_{q}(z, T z) \leq F\left(d_{q}(z, T z), \varphi\left(d_{q}(z, T z)\right)\right)
$$

so,

$$
d_{q}(z, T z)=0 \quad \text { or } \quad \varphi\left(d_{q}(z, T z)\right)=0
$$

therefore, we obtain $d_{q}(z, T z)=0$. Again, from (2.18) we get $d_{q}(T z, z)=0$. As a result $z=T z$.

Uniqueness, Let us suppose that $u$ and $v$ are two fixed points of $T$ where $T u=u$ and $T v=v$.

From the condition of the theorem we have,

$$
\begin{array}{r}
d_{q}(T u, T v) \leq F\left(g\left[d_{q}(u, v), d_{q}(u, T u), d_{q}(v, T v)\right],\right. \\
\left.\varphi\left(g\left[d_{q}(u, v), d_{q}(u, T u), d_{q}(v, T v)\right]\right)\right) .
\end{array}
$$

If we replace $v=u$ in (2.19) then we obtain,

$$
\begin{aligned}
d_{q}(u, u)= & d_{q}(T u, T u) \\
\leq & F\left(g\left[d_{q}(u, u), d_{q}(u, T u), d_{q}(u, T u)\right],\right. \\
& \left.\varphi\left(g\left[d_{q}(u, u), d_{q}(u, T u), d_{q}(u, T u)\right]\right)\right) \\
= & F\left(g\left[d_{q}(u, u), d_{q}(u, u), d_{q}(u, u)\right], \varphi\left(g\left[d_{q}(u, u), d_{q}(u, u), d_{q}(u, u)\right]\right)\right) \\
\leq & F\left(d_{q}(u, u), \varphi\left(d_{q}(u, u)\right)\right) .
\end{aligned}
$$


Thus $d_{q}(u, u)=0$ or $\varphi\left(d_{q}(u, u)\right)=0$, therefore we get $d_{q}(u, u)=0$. Similarly we have that $d_{q}(v, v)=0$. Therefore, using the condition (2.18) we have:

$$
\begin{aligned}
d_{q}(u, v) & =d_{q}(T u, T v) \\
& \leq F\left(g\left[d_{q}(u, v), d_{q}(u, u), d_{q}(v, v)\right], \varphi\left(g\left[d_{q}(u, v), d_{q}(u, u), d_{q}(v, v)\right]\right)\right) \\
& \leq F\left(d_{q}(u, v), \varphi\left(d_{q}(u, v)\right)\right) .
\end{aligned}
$$

And also,

$$
\begin{aligned}
d_{q}(v, u) & =d_{q}(T v, T u) \\
& \leq F\left(g\left[d_{q}(v, u), d_{q}(v, v), d_{q}(u, u)\right], \varphi\left(g\left[d_{q}(v, u), d_{q}(v, v), d_{q}(u, u)\right]\right)\right) \\
& \leq F\left(d_{q}(v, u), \varphi\left(d_{q}(v, u)\right)\right) .
\end{aligned}
$$

So $d_{q}(u, v)=0$ or $\varphi\left(d_{q}(u, v)\right)=0$, and $d_{q}(v, u)=0$ or $\varphi\left(d_{q}(v, u)\right)=0$, from these two we have $d_{q}(u, v)=d_{q}(v, u)=0$, and the property $d_{2}$ implies $v=u$. Hence, the fixed point is unique.

Taking $F(s, t)=k s, 0<k<1$, in Theorem 2.1 we have the following remark

Remark 2.1. [22] Let $K$ and $H$ be nonempty subsets of a complete dislocated quasimetric space $\left(X, d_{q}\right)$. Let $T: K \cup H \rightarrow K \cup H$ be a cyclic mapping that satisfies the condition

$$
d_{q}(T x, T y) \leq k \max \left\{d_{q}(x, y), d_{q}(x, T x), d_{q}(y, T y)\right\}
$$

for all $x \in K$ and $y \in H$ and $0 \leq k<1$.Then, $T$ has a unique fixed point in $K \cap H$.

Taking $F(s, t)=\beta(s) s$, in Theorem 2.2 we have the following remark

Remark 2.2. [22] Let $K$ and $H$ be nonempty closed subsets of a dislocated quasi-metric space $\left(X, d_{q}\right)$ and $T: K \cup H \rightarrow K \cup H$ be a cyclic mapping that satisfies the Geraghty type condition:

$$
d_{q}(T x, T y) \leq \beta\left(d_{q}(x, y)\right) d_{q}(x, y)
$$

for all $x \in K$ and $y \in H$ where $\beta \in S$. Then $T$ has a unique fixed point in $K \cap H$.

Taking $F(s, t)=k s, 0<k<1$, in Theorem 2.3 we have the following remark

Remark 2.3. [22] Let $K$ and $H$ be nonempty closed subsets of a dislocated quasimetric space $(X, d)$ and $T: K \cup H \rightarrow K \cup H$ be a cyclic mapping that satisfies the following condition:

$$
d_{q}(T x, T y) \leq k g\left[d_{q}(x, y), d_{q}(x, T x), d_{q}(y, T y)\right]
$$

for all $x \in K$ and $y \in H$, and $0<k<1$, where $g \in G_{3}$. Then $T$ has a unique fixed point in $K \cap H$. 
Example 2.2. Let $X=[-1,1]$ and $T: X \rightarrow X$ be given as $T x=\frac{-x}{5}$. Let $K=[-1,0]$ and $H=[0,1]$. Define the function $d_{q}: X \times X \rightarrow[0, \infty)$ by $d_{q}(x, y)=|x-y|+|x|$. We note that $d_{q}$ is a dislocated quasi-metric on $X$ and the map $T$ is cyclic on $X$.

If we consider from $G_{3}$ the function $g\left(t_{1}, t_{2}, t_{3}\right)=\max \left\{t_{1}, t_{2}, t_{3}\right\}$ we see

$$
\begin{aligned}
d_{q}(x, y) & =|x-y|+|x| \\
d_{q}(T x, T y) & =\left|\frac{-x}{5}-\frac{-y}{5}\right|+\left|\frac{-x}{5}\right|=\frac{1}{5}|x-y|+\frac{1}{5}|x| .
\end{aligned}
$$

Then, clearly, we have,

$$
\begin{aligned}
d_{q}(T x, T y)= & \left|\frac{-x}{5}-\frac{-y}{5}\right|+\left|\frac{-x}{5}\right| \\
= & \frac{1}{5}(|x-y|+|x|)=\frac{1}{5} d(x, y) \\
& \leq k g\left[d_{q}(x, y), d_{q}(x, T x), d_{q}(y, T y)\right] .
\end{aligned}
$$

So for the constant $\frac{1}{5} \leq k<1$ the map $T$ satisfies the condition (2.18) of Theorem 2.3 and 0 is a unique fixed point of $T$.

From the general character of Theorems 2.1, 2.2, and 2.3, and because of the class $C$ and $G_{3}$, we can give many corollaries as follows using the functions

$$
\begin{aligned}
& g\left(t_{1}, t_{2}, t_{3}\right)=\left[\max \left\{t_{1}^{p}, t_{2}^{p}, t_{3}^{p}\right\}\right]^{\frac{1}{p}}, p>0 \\
& g\left(t_{1}, t_{2}, t_{3}\right)=\left[\max \left\{t_{1} t_{2}, t_{2} t_{3}, t_{1} t_{3}\right\}\right]^{\frac{1}{2}}, p>0 \\
& g\left(t_{1}, t_{2}, t_{3}\right)=g \max \left\{t_{1}+t_{2}, t_{2}+t_{3}, t_{1}+t_{3}\right\} .
\end{aligned}
$$

Corollary 2.1. Let $K$ and $H$ be nonempty closed subsets of a dislocated quasimetric space $(X, d)$ and $T: K \cup H \rightarrow K \cup H$ be a cyclic mapping that satisfies the following condition:

$$
d^{p}(T x, T y) \leq k \max \left\{d^{p}(x, y), d^{p}(x, T x), d^{p}(y, T y)\right\}
$$

for all $x \in K$ and $y \in H$, and $0 \leq k<1$. Then $T$ has a unique fixed point in $K \cap H$.

Proof. Taking $F(s, t)=k s, 0<k<1$ and $g\left(t_{1}, t_{2}, t_{3}\right)=\left[\max \left\{t_{1}^{p}, t_{2}^{p}, t_{3}^{p}\right\}\right]^{\frac{1}{p}}, p>0$ in Theorem 2.3 .

Corollary 2.2. Let $K$ and $H$ be nonempty closed subsets of a dislocated quasimetric space $\left(X, d_{q}\right)$ and $T: K \cup H \rightarrow K \cup H$ be a cyclic mapping that satisfies the following condition:

$d_{q}{ }^{2}(T x, T y) \leq k \max \left\{d_{q}(x, y) d_{q}(x, T x), d_{q}(x, y) d_{q}(y, T y), d_{q}(x, T x) d_{q}(y, T y)\right\}$ for all $x \in K$ and $y \in H$, and $0 \leq k<1$. Then $T$ has a unique fixed point in $K \cap H$.

Proof. Taking $F(s, t)=k s, 0<k<1$ and $g\left(t_{1}, t_{2}, t_{3}\right)=\left[\max \left\{t_{1} t_{2}, t_{2} t_{3}, t_{1} t_{3}\right\}\right]^{\frac{1}{2}}$, $p>0$ in Theorem 2.3. 
Corollary 2.3. Let $K$ and $H$ be nonempty closed subsets of a dislocated quasimetric space $\left(X, d_{q}\right)$ and $T: K \cup H \rightarrow K \cup H$ be a cyclic mapping that satisfies the following condition:

$$
\begin{aligned}
d_{q}(T x, T y) \leq k \max \{ & d_{q}(x, y)+d_{q}(x, T x), d_{q}(x, y) \\
& \left.+d_{q}(y, T y), d_{q}(x, T x)+d_{q}(y, T y)\right\}
\end{aligned}
$$

for all $x \in K$ and $y \in H$, and $0 \leq k<1$. Then $T$ has a unique fixed point in $K \cap H$.

Proof. Taking $F(s, t)=k s, 0<k<1$ and $g\left(t_{1}, t_{2}, t_{3}\right)=\max \left\{t_{1}+t_{2}, t_{2}+t_{3}, t_{1}+t_{3}\right\}$, $p>0$ in Theorem 2.3.

Further, as common applications of fixed point theorems we provide some corollaries for cyclic maps for integral type contraction (taking $K=H=X$ ).

Corollary 2.4. Let $\left(X, d_{q}\right)$ be a complete dislocated quasi-metric space and $T$ : $X \rightarrow X$ be a mapping such that for any $x, y \in X$,

$$
\int_{0}^{d_{q}(T x, T y)} \rho(t) d t \leq r \int_{0}^{\beta\left(d_{q}(x, y)\right) d_{q}(x, y)} \rho(t) d t
$$

where the function $\phi \in \Phi$, the constant $r \in[0,1)$ and $\rho:[0, \infty) \rightarrow[0, \infty)$ is Lesbegueintegrable mapping satisfying $\int_{0}^{\varepsilon} \rho(t) d t>0$ for $\varepsilon>0$. Then, $T$ has a unique fixed point in $X$.

Remark 2.4. Theorem 2.3 generalizes and unifies the results for the Kannan-type cyclic contraction, Chatterjea cyclic contraction, $C$-cyclical contraction, Zamfirescu contraction and some existing results in dislocated-metric spaces $[1,5,23,24]$. Statements of many theorems and results can be obtained by taking $K=H=X$.

\section{REF E R E N C E S}

1. C. T. Aage and J. N. Salunke. The results on fixed points in dislocated and dislocated quasi-metric space. Appl. Math. Sci., 2 (2008):2941-2948.

2. A. H. Ansari, Note on " $\varphi-\psi$-contractive type mappings and related fixed point", The 2nd Regional Conference on Mathematics And Applications, Payame Noor University, September 2014, 377-380.

3. Z.M. Fadail, A.G. Bin Ahmad, A. H. Ansari, S. Radenovic, M. Rajović, Some Common Fixed Point Results of Mappings in 0-Complete Metric-like Spaces via New Function, Applied Mathematical Sciences, Vol. 9, 2015, no. 83, 4109-4127.

4. M. A. Geraghty, On contractive mappings, Proc. Amer. Math. Soc. 40(1973), 604-608.

5. R. George, R. Rajagopalan, S. Vinayagam. Cyclic contractions and fixed points in dislocated metric spaces. Int. Journal of Math. Analysis, vol. 7, 2013, no.9, 403-411.

6. P. Hitzler and A. K. Seda. Dislocated topologies. J. Electr. Engin., 51(12/S):3:7, 2000.

7. P. Hitzler. Generalized Metrics and Topology in Logic Programming Semantics. Ph.D. thesis, National University of Ireland, University College Cork, 2001. 
8. E. Hoxha, A. H. Ansari, K. Zoto, Some common fixed point results through generalized altering distances on dislocated metric spaces, Proceedings of EIIC, September 1-5, 2014, 403-409.

9. K. Jha and D. Panthi, A Common Fixed Point Theorem in Dislocated Metric Space, Appl. Math. Sci., vol. 6, 2012, no. 91, 4497-4503.

10. E. Karapinar and I. M Erhan, Best Proximity on Different Type Contractions Applied Mathematics and Information Science, 2010.

11. E. Karapinar and H. K. Nashine, Fixed point Theorems for Kannan type cyclic weakly contractions Journal of Nonlinear Analysis and Optimization, vol. 4, N0. 1, (2013), 2935.

12. E. Karapinar, Fixed point Theory for cyclic weak $\phi$-contraction, Appl. Math. Lett. 24 (2011) 822-825.

13. E. Karapinar and P. Salimi, Dislocated metric space to metric spaces with some fixed point theorems, Fixed point theory and applications (2013).

14. S. Karpagam and S. Agrawal, (2010) Best Proximity Points theorems for Cyclic Meir Keeler Contraction Maps, Nonlinear Analysis doi:10.1016/j.na.2010.07.26 (in press)

15. M. S. Khan, M. Swaleh, and S. Sessa, Fixed point theorems by altering distances between the points, Bulletin of the Australian Mathematical Society, 30 (1) (1984) $1-9$.

16. W.A.Kirk and P.S. Srinivasan and P.Veeramani. Fixed Points for mapping satisfying Cyclic contractive conditions. Fixed Point Theory, 4 (2003), 79-89.

17. Ch. Klin-eam and Ch. Suanoom, Dislocated quasi-b-metric spaces and fixed point theorems for cyclic contractions, Fixed Point Theory and Applications, 2015, 2015:74.

18. Kumari, P. Sumati, and D. Panthi. "Cyclic contractions and fixed point theorems on various generating spaces." Fixed Point Theory and Applications, 2015. 1 (2015): 153.

19. Kumari, P. Sumati, and D. Panthi. "Connecting various types of cyclic contractions and contractive self-mappings with Hardy-Rogers self mappings." Fixed Point Theory and Applications, 2016, 1 (2016): 1-19.

20. Kumari, P. Sumati. "On dislocated quasi metrics." Journal of Advanced Studies in Topology 3.2 (2012): 66-75.

21. F. M. Zeyada, G. H. Hasan and M. A. Ahmed, A generalization of a fixed point theorem due to Hitzler and Seda in dislocated quasi-metric spaces, Arab. J. Sci. Eng. Sec. A Sci., 31(2006), 111-114.

22. K. Zoto, P.S. Kumari, E. Hoxha, Some Fixed Point Theorems and Cyclic Contractions in Dislocated and Dislocated Quasi-Metric Spaces,American Journal of Numerical Analysis, 2014, Vol. 2, No. 3, 79-84.

23. K. Zoto and E. Hoxha, Fixed point theorems in dislocated and dislocated quasi-metric space Journal of Advanced Studies in Topology; Vol. 3, No.1, 2012.

24. K. Zoto and E. Hoxha, Fixed point theorems for cyclic contractions. Proceedings in ARSA, the second conference of advanced research in scientific areas. 2-6 December 2013. 
Arslan Hojat Ansari

Islamic Azad University, Karaj Branch

Department of Mathematics

Karaj, Iran

analsisamirmath2@gmail.com

Kastriot Zoto

University of Gjirokastra

Faculty of Natural Sciences

Department of Mathematics and Informatics

Gjirokatra, Albania

zotokastriot@yahoo.com 